\title{
Carbon Dot Based Sensing of Dopamine and Ascorbic Acid
}

\author{
Upama Baruah, ${ }^{1}$ Neelam Gogoi, ${ }^{1}$ Achyut Konwar, ${ }^{1}$ Manash Jyoti Deka, ${ }^{1}$ \\ Devasish Chowdhury, ${ }^{1}$ and Gitanjali Majumdar ${ }^{2}$ \\ ${ }^{1}$ Material Nanochemistry Laboratory, Institute of Advanced Study in Science and Technology, Paschim Boragaon, Garchuk, \\ Guwahati 781035, India \\ ${ }^{2}$ Department of Chemistry, Assam Engineering College, Jalukbari, Guwahati 781035, India
}

Correspondence should be addressed to Devasish Chowdhury; devasishc@gmail.com and Gitanjali Majumdar; gitanjalic@gmail.com

Received 29 August 2014; Revised 3 December 2014; Accepted 3 December 2014; Published 29 December 2014

Academic Editor: Shu Taira

Copyright (C) 2014 Upama Baruah et al. This is an open access article distributed under the Creative Commons Attribution License, which permits unrestricted use, distribution, and reproduction in any medium, provided the original work is properly cited.

We demonstrate carbon dot based sensor of catecholamine, namely, dopamine and ascorbic acid. Carbon dots (CDs) were prepared from a green source: commercially available Assam tea. The carbon dots prepared from tea had particle sizes of $\sim 0.8 \mathrm{~nm}$ and are fluorescent. Fluorescence of the carbon dots was found to be quenched in the presence of dopamine and ascorbic acid with greater sensitivity for dopamine. The minimum detectable limits were determined to be $33 \mu \mathrm{M}$ and $98 \mu \mathrm{M}$ for dopamine and ascorbic acid, respectively. The quenching constants determined from Stern-Volmer plot were determined to be $5 \times 10^{-4}$ and $1 \times 10^{-4}$ for dopamine and ascorbic acid, respectively. A probable mechanism of quenching has been discussed in the paper.

\section{Introduction}

Carbon dots are recently being used as one of the most efficient and stable nanoprobes for various types of analytes owing to their easy preparation, photostability, and low toxicity compared to other heavy metals containing quantum dots $[1,2]$. They belong to the family of carbon nanomaterials and show characteristic bright blue or green fluorescence under UV light irradiation. They show higher degrees of biocompatibility and hence are most suitable for in vivo applications also. Hence, there are a growing number of reports for easy synthesis methods using easily available sources like orange juice [3], bread [4], plant leaf [5], dopamine [6], polyethylene glycol [7, 8], chitosan [9], and tea [10]. The fluorescence properties of these carbon dots have been utilized for efficient sensing of a wide variety of biomolecules. For example, Lin et al. [11] have used the chemiluminescence property of the carbon dots for sensing of nitrate. Lu and coworkers have used carbon nanoparticles prepared from pomelo peel, for the detection of mercury ions [12]. In the past, our laboratory had also demonstrated the use of carbon dots capped with calixarenes as fluoride [13] and hemin sensors [14].

Among the various biomolecules, catecholamines are a very important class of neurotransmitters, for their very important role in various vital functions of our body. Any deficiency of these biomolecules leads to serious neurological disorders like Parkinson, Schizophrenia, and other serious renal and cardiovascular diseases. Hence, their sensing is very important to maintain proper human health. Various methods are reported till today for the sensing of the biomolecules using carbon nanomaterials, which are basically based on electroactive properties of the nanomaterials. For example Zhao et al. [15] have used carbon nanotubes in three-dimensional nanoweb structured electrode for highly selective detection of dopamine in presence of ascorbic acid. But very few reports are there for using carbon nanomaterials for optical sensing of these neurotransmitters. Optical sensing based methods are more convenient as they are very fast, noninvasive, and highly sensitive. The fluorescence properties of carbon nanomaterials can be utilized for sensing of these neurotransmitters, specially dopamine and ascorbic 
acid. To the best of our knowledge, such fluorescence based techniques using carbon nanomaterials are very limited in case of these neurotransmitters. Among these very limited numbers of attempts, $\mathrm{Qu}$ and coworkers demonstrated the use of carbon dots for the detection of iron and dopamine [6]. Tao et al. demonstrated a dual fluorometric and colorimetric sensor for dopamine based on BSA-stabilized Au nanoclusters [16]. In more recent work by Zhou et al. [17], polypyrrole/graphene quantum dots core-shell hybrids have been used for the fluorimetric sensing of dopamine up to nanomolar concentration.

In this present work, we have designed a very simple system, based on carbon dots for the sensing of dopamine and ascorbic acid in aqueous medium. Fluorescence of the carbon dots was found to be quenched in the presence of both analytes with greater sensitivity for dopamine. Carbon dots were prepared via greener method from Assam Tea developed in our lab. A possible mechanism is also proposed for the observed result.

\section{Experimental Section}

2.1. Materials and Instruments. The commercially available Assam tea was used as received. Dopamine hydrochloride was purchased from Sigma Aldrich. Glacial acetic acid and ascorbic acid ( $\geq 99 \%)$ were purchased from Merck. All reagents were used as received without further purification. The water used was from a Milli-Q water purification system.

Particle size and zeta potential measurements were done on a Malvern Zetasizer Nanoseries, Nano-ZS90. SEM images were collected on a Carl Zeiss $\sum$ igma VP instrument. Powder XRD spectrum was collected on a Bruker D8 Advance diffractometer. UV-Visible absorption spectra were collected on a Shimadzu UV-VIS Spectrophotometer, UV-2600, and fluorescence spectra were collected on a Varian Cary Eclipse Spectrofluorometer. Fourier transform infrared spectra were collected on a Nicolet-6700 FT-IR Spectrophotometer.

2.2. Preparation of Carbon Dots (CDs) from Tea. Carbon dots from tea were prepared according to a method developed and reported by our laboratory [10]. Briefly, commercially available Assam tea was first heated at $200^{\circ} \mathrm{C}$ for about 2 hours, followed by grinding to powdered form and again heating at $200^{\circ} \mathrm{C}$ for about 8 hours. The so formed black carbonized powder of tea was cooled to room temperature and stored in a glass vial. $0.1 \mathrm{M}$ acetic acid was used as the dispersion medium for the preparation of CDs from tea. A quantity of $300 \mathrm{mg}$ of the carbonized tea was dispersed in $10 \mathrm{~mL}$ of $0.1 \mathrm{M}$ acetic acid and kept for 40 hours. The dispersed medium was then centrifuged (at 10,000 rpm for $0.5 \mathrm{~h}$ ) and the supernatant liquid containing tea CDs was collected and preserved.

2.3. Fluorescence Quenching of Tea Carbon Dots by Dopamine and Ascorbic Acid. Appropriate amounts of dopamine and ascorbic acid were added to the carbon dot solution and the corresponding fluorescence spectra were recorded.
The solution was stirred vigorously every time before recording the PL spectra.

\section{Results and Discussion}

Carbon dots from tea were prepared according to a method developed and reported by our laboratory [10]. This carbon dot solution was then used as a material for optical sensing of dopamine and ascorbic acid as shown in Scheme 1.

3.1. Characterization of Carbon Dots Prepared from Tea. The carbon dots prepared from tea were characterized by dynamic light scattering (DLS) technique, scanning electron microscopy (SEM), powder XRD measurement, UVVisible absorption spectroscopy, fluorescence spectroscopy, and Fourier transform infrared spectroscopy. The particle size of CDs was determined by dynamic light scattering (DLS) measurement (Figure 1(a)). The DLS data showed the particle size of CDs to be $\sim 0.8 \mathrm{~nm}$. The zeta potential of CDs was also measured and was found to be $-184 \mathrm{mV}$ indicating the negative charge on the CDs. The particle sizes of CDs were also determined by SEM (Figure 1(b)).

CDs prepared from tea being highly fluorescent materials, photoluminescence properties were investigated. Figure 2(a) shows the UV-VIS absorption and photoluminescence (PL) spectra of CDs excited at $340 \mathrm{~nm}$. The UV-VIS absorption spectrum of CDs shows two distinct peaks, one at around $300 \mathrm{~nm}$ that can be assigned to $n-\pi^{*}$ transition of the $\mathrm{O}-\mathrm{H}$ groups present on the surface of CDs and the other peak appearing at around $250 \mathrm{~nm}$ that can be assigned to $\pi-\pi^{*}$ transitions of the aromatic systems in the polyphenols of tea. The CD solution showed maximum emission of PL intensity at an excitation wavelength of $340 \mathrm{~nm}$. For a $\lambda_{\text {exc }}$ value of $340 \mathrm{~nm}$, the CD solution exhibits a $\lambda_{\mathrm{em}}$ value of $446 \mathrm{~nm}$. The inset of Figure 2(a) shows the images of the CD solution viewed under normal light and UV light.

Figure 2(b) shows the powder XRD spectrum of carbon dots prepared from tea. The powder XRD spectrum shows a sharp peak at around $2 \theta=30^{\circ}$ which indicates that the prepared carbon dots are crystalline in nature. Figure 2(c) shows the FTIR spectrum of CDs prepared from tea. The peak at $3418 \mathrm{~cm}^{-1}$ can be assigned to $\mathrm{O}-\mathrm{H}$ stretching vibration of the hydroxyl group present in the different types of polyphenols present in tea. The peaks appearing at 1642 and $1599 \mathrm{~cm}^{-1}$ can be assigned to C-C stretch (in-ring) of the polyphenols. Peaks at 1470 and $1388 \mathrm{~cm}^{-1}$ can be assigned to $\mathrm{C}-\mathrm{H}$ bend and $\mathrm{C}-\mathrm{H}$ rock stretching vibrations, respectively. Peaks at 1350 and $1117 \mathrm{~cm}^{-1}$ can be assigned to $\mathrm{C}-\mathrm{O}$ stretching vibrations of phenols and ether groups in the polyphenolic structures. Peaks at 766 and $618 \mathrm{~cm}^{-1}$ can be assigned to $\mathrm{C}-\mathrm{H}$ in the aromatic structures.

3.2. Detection of Dopamine by the Carbon Dots. The carbon dots prepared from tea were successfully used for sensing of dopamine. A very simple and facile optical detection method, that is, fluorescence spectroscopy, was used to monitor the detection of dopamine by the tea carbon dots. Figures 3(a) and 3(b) show the stacked photoluminescence (PL) spectra of 


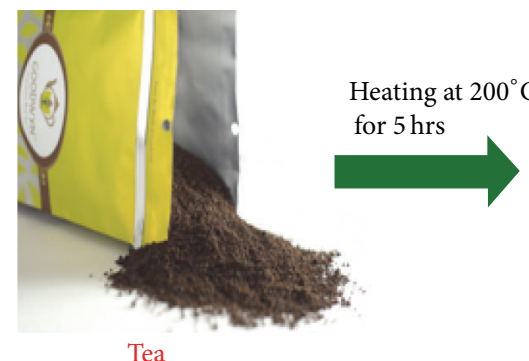

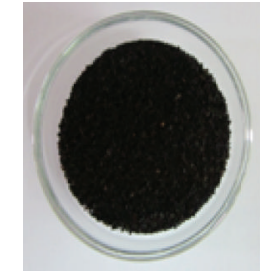

Calcinated tea

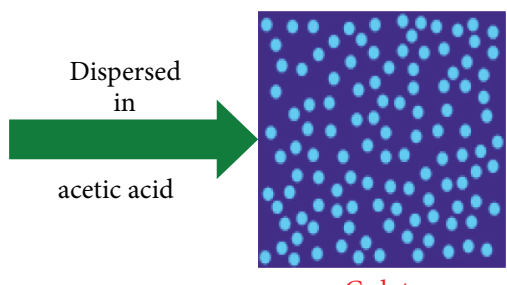

C-dot

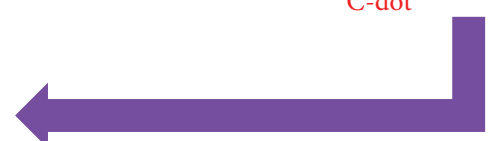<smiles>NCCc1ccc(O)c(O)c1</smiles>

Dopamine

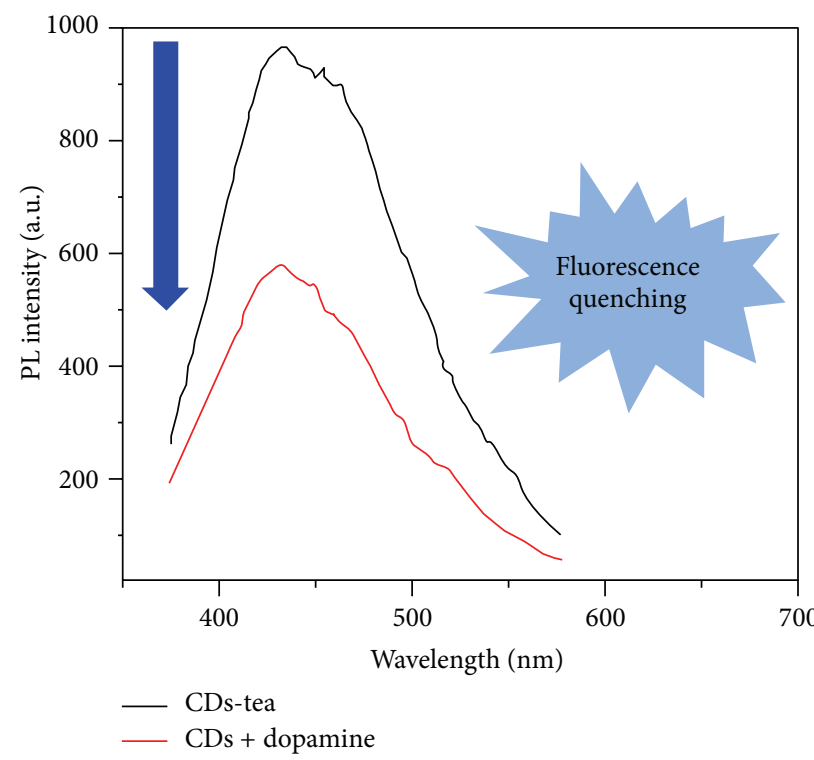

Scheme 1: Schematic representation of the method of preparation of carbon dots from tea and the optical response of this material to addition of dopamine monitored by fluorescence spectroscopy.

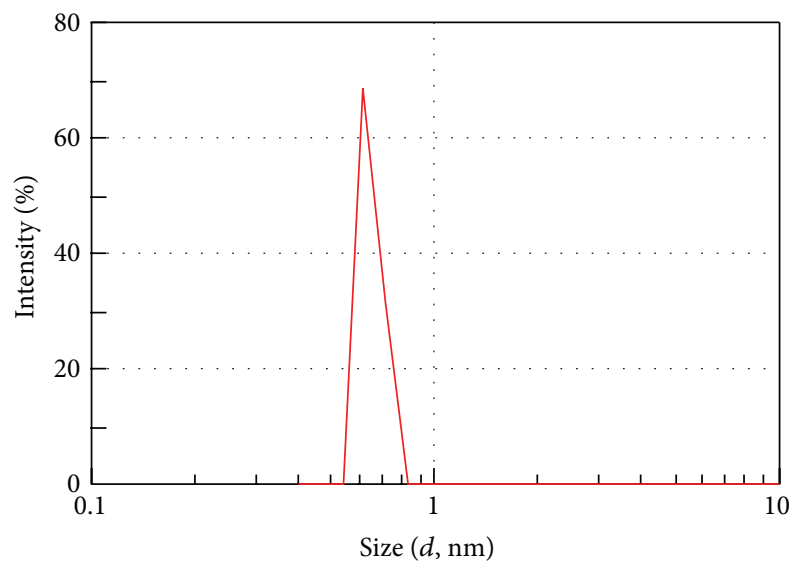

(a)

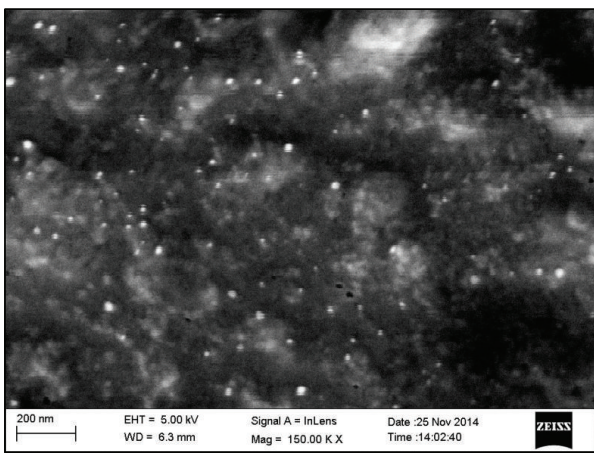

(b)

Figure 1: Particle size of CDs prepared from tea as obtained from (a) dynamic light scattering (DLS) technique and (b) scanning electron microscopy (SEM).

CDs upon addition of different concentrations of dopamine and ascorbic acid, respectively. It is evident from Figure 3(a) that addition of dopamine results in gradual decrease in emission intensity of the CDs studied in the concentration range $\sim 33 \mu \mathrm{M}-1250 \mu \mathrm{M}$. Similar behavior in fluorescence intensity is observed for CDs upon addition of ascorbic acid. However, in case of ascorbic acid, the concentration range is $\sim 98 \mu \mathrm{M}-1250 \mu \mathrm{M}$.

As evident from Figures 3(a) and 3(b), the amount of quenching of fluorescence intensity of CDs by dopamine is 


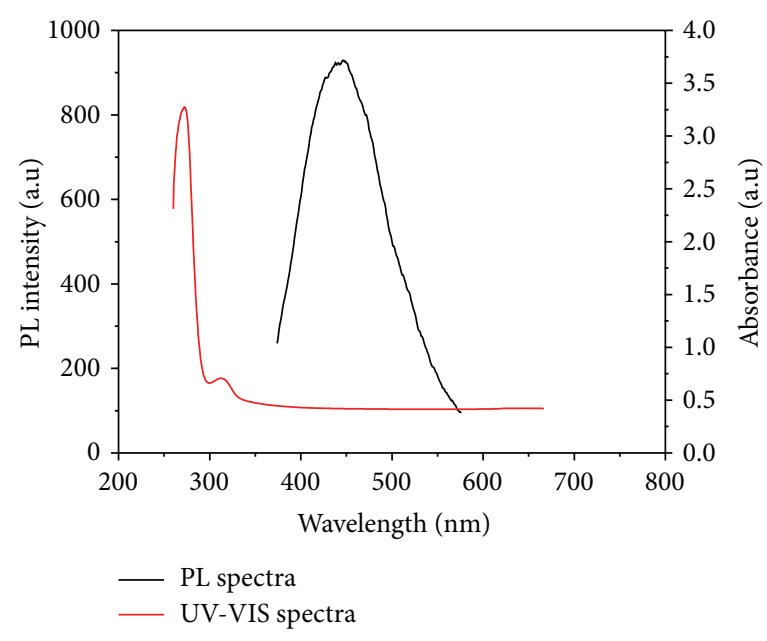

(a)

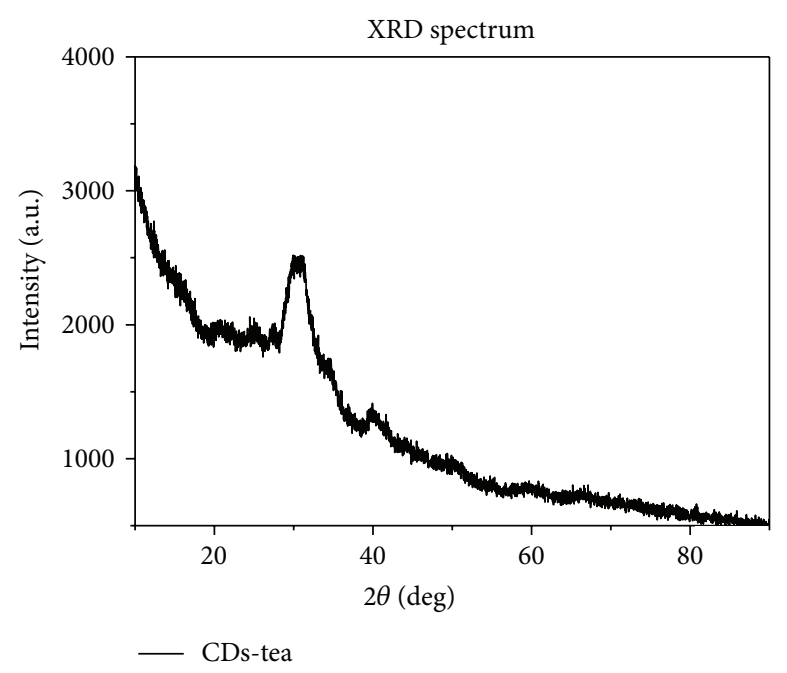

(b)

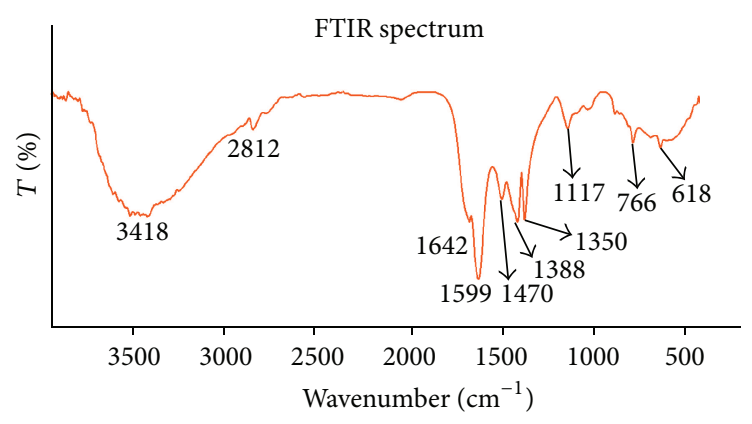

(c)

Figure 2: (a) The UV-VIS absorption spectrum and photoluminescence (PL) spectrum of CDs excited at $340 \mathrm{~nm}$. The inset of (a) shows the images of the CD solution viewed under normal light and UV light. (b) Powder XRD spectrum of CD solution and (c) FTIR spectrum of CDs prepared from tea.

greater than that observed with ascorbic acid. To determine the fluorescence quenching efficiency of dopamine and ascorbic acid, the quenching data was fitted to the Stern-Volmer equation given by

$$
\frac{F_{0}}{F}=1+K[Q],
$$

where $F_{0}$ and $F$ are the fluorescence quantum efficiencies in the absence and presence of quencher, respectively, $K$ is the Stern-Volmer quenching constant, and [Q] is the quencher concentration. Figures 4(a) and 4(b) show the linear graphs obtained by fitting the quenching data into (1).

As evident from Figure 4(a), a good linear relationship could be obtained for dopamine in the concentration range $33 \mu \mathrm{M}-1250 \mu \mathrm{M}$. The value of $K$ as obtained from the SternVolmer plot for dopamine is $5 \times 10^{-4}$ and the value of correlation coefficient was found to be 0.9815 . Similarly, from Figure 4(b), it is evident that linear relationship could be obtained for ascorbic acid in the concentration range $98 \mu \mathrm{M}-1250 \mu \mathrm{M}$. The value of $K$ as obtained from the SternVolmer plot for ascorbic acid is $1 \times 10^{-4}$ and the value of correlation coefficient was found to be 0.9363 . Therefore, from Figures 3 and 4, it is quite clear that dopamine is a much more efficient quencher of the carbon dot fluorescence as compared to ascorbic acid. Further, from (1), it is seen that, with all other terms held constant, the higher the value of $K$, the lower the concentration of the quencher required to achieve the fluorescence quenching. In our case, this fact is very much evident from Figures 3(a) and 3(b). That is, the minimum amount of dopamine required to cause the fluorescence quenching of CDs is $33 \mu \mathrm{M}$, whereas, under the exactly same conditions, the amount of ascorbic acid required to cause the fluorescence quenching of CDs is $98 \mu \mathrm{M}$. This fact clearly demonstrates the sensitivity of the tea carbon dots for dopamine.

In order to study the effect of the acetic acid medium or the interference caused by the ascorbic acid molecules, we performed the quenching experiment of carbon dots with a mixture of dopamine and ascorbic acid and it is observed that the fluorescence quenching of CDs occurred even for the mixture. The data obtained from the SternVolmer equation was then fitted into linear plots against 

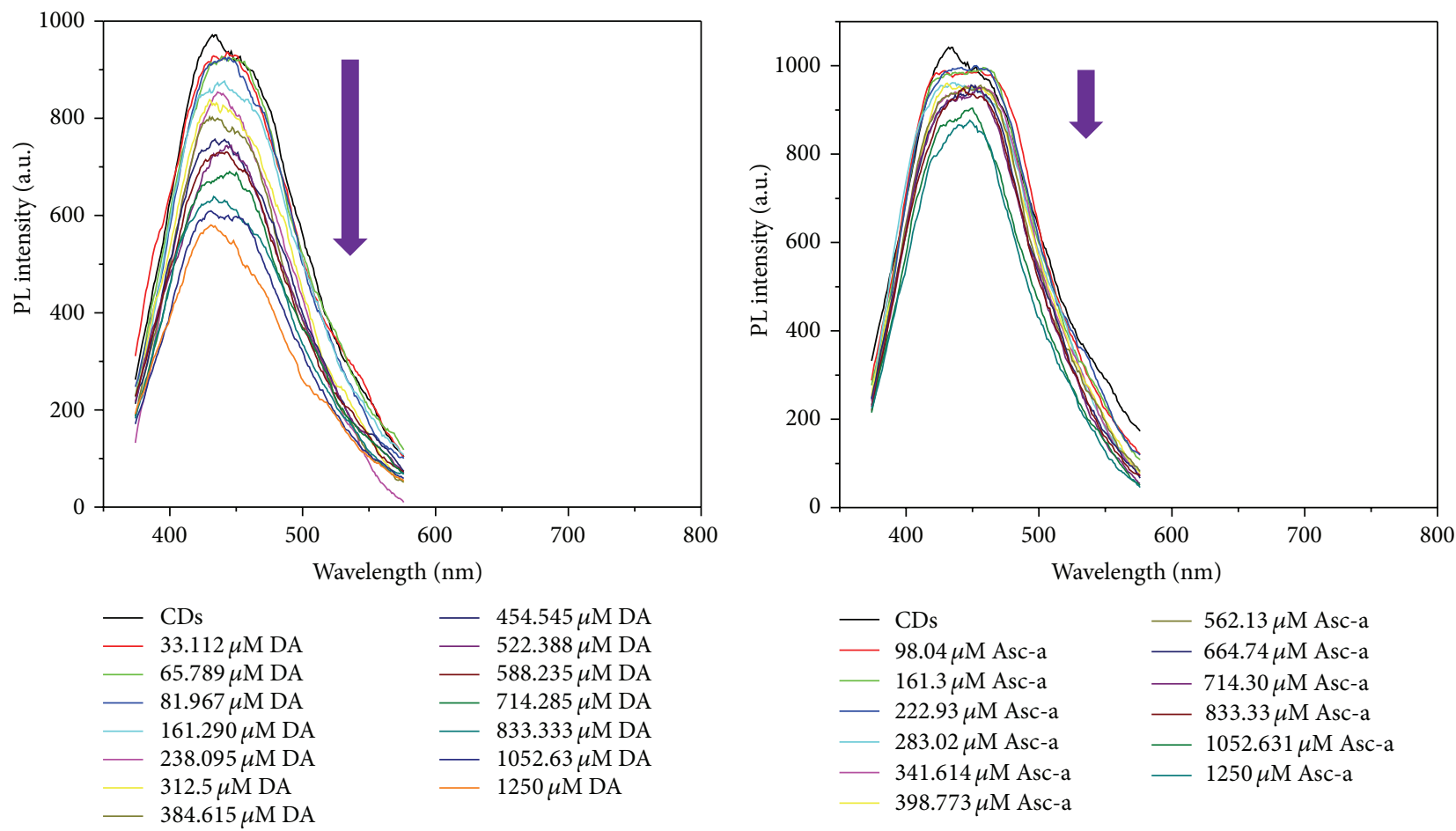

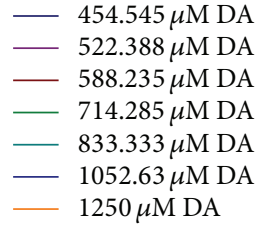

(a)

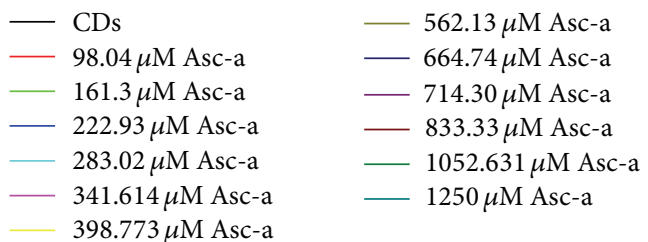

(b)

FIGURE 3: The stacked photoluminescence (PL) spectra of CDs upon addition of different concentrations of (a) dopamine and (b) ascorbic acid.

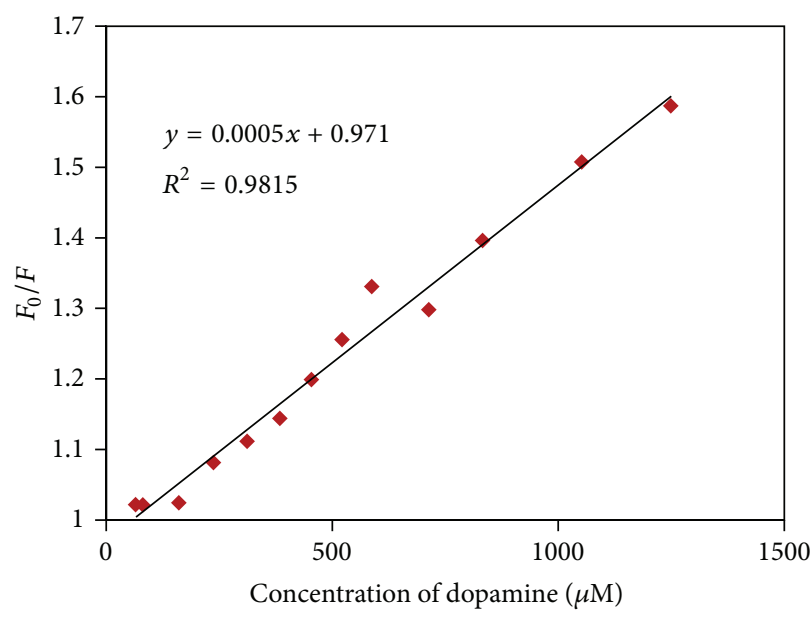

(a)

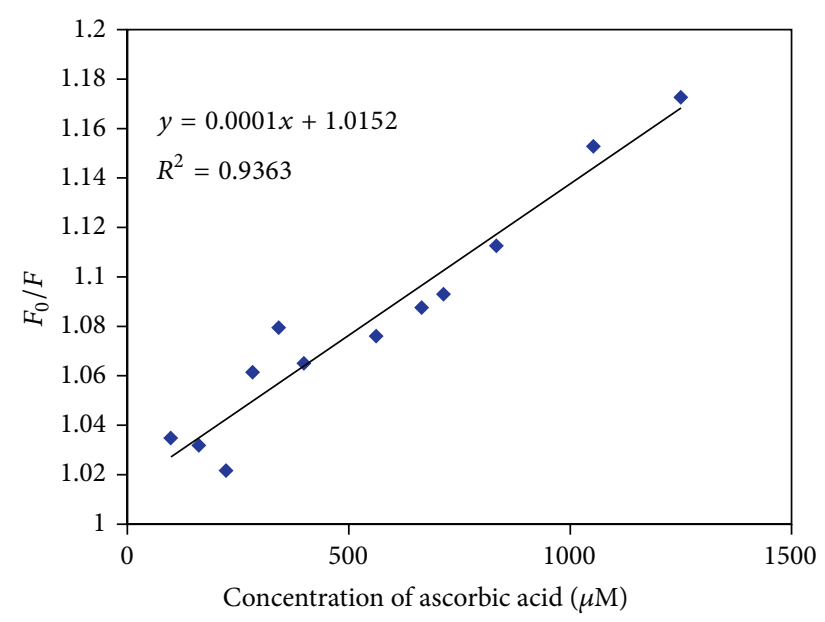

(b)

FIgURE 4: The linear fitted results for (a) dopamine and (b) ascorbic acid using Stern-Volmer equation.

different concentrations of the mixture. From the plot, it is clear that the data can be fitted into two different linear patterns with different slopes, that is, quenching efficiencies. Figure 5(a) shows the stacked photoluminescence (PL) spectra of CDs upon addition of different concentrations of a mixture of dopamine and ascorbic acid in the concentration range $40-400 \mu \mathrm{M}$. Figure $5(\mathrm{~b})$ shows the linear fitted results for different concentrations of the mixture of dopamine and ascorbic acid showing two different linear fitting patterns.
The value of $K$ was then calculated for the mixture from the two linear fitted graphs as shown in Figure 5(b). $K$ values obtained from the Stern-Volmer plot for the two linear fitted graphs were obtained as $6 \times 10^{-4}$ and $2 \times 10^{-4}$, respectively. The correlation coefficients were determined to be 0.9354 and 0.9841 , respectively. The $K$ value was already calculated for pure dopamine and ascorbic acid (Figure 4), so it is evident that first linear plot is for dopamine and second plot is for ascorbic acid. Hence, even when ascorbic acid and dopamine 


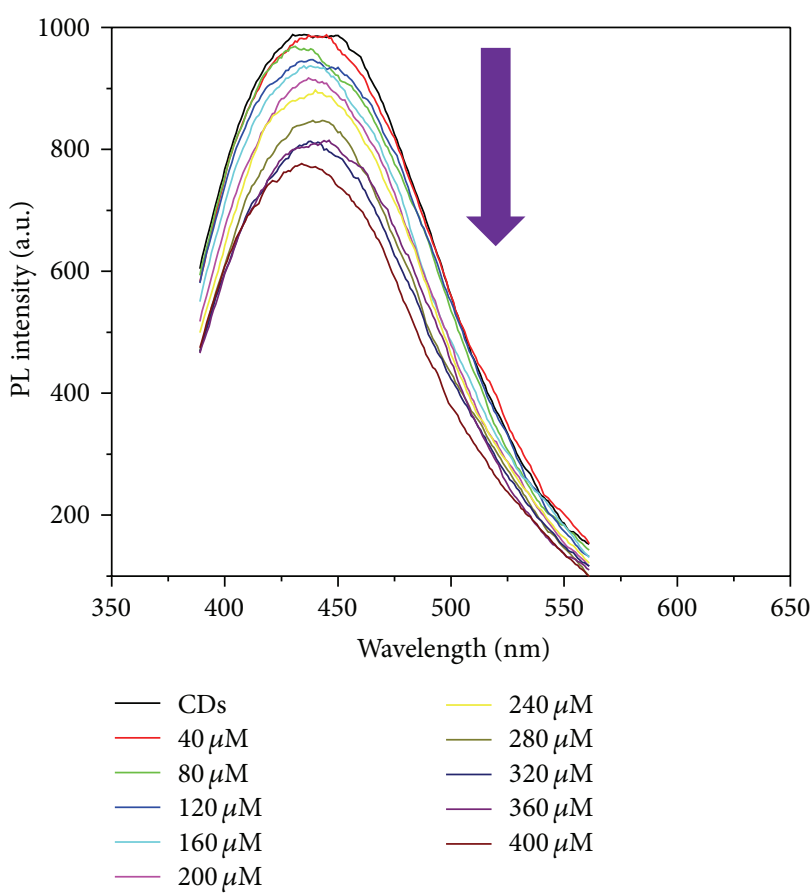

(a)

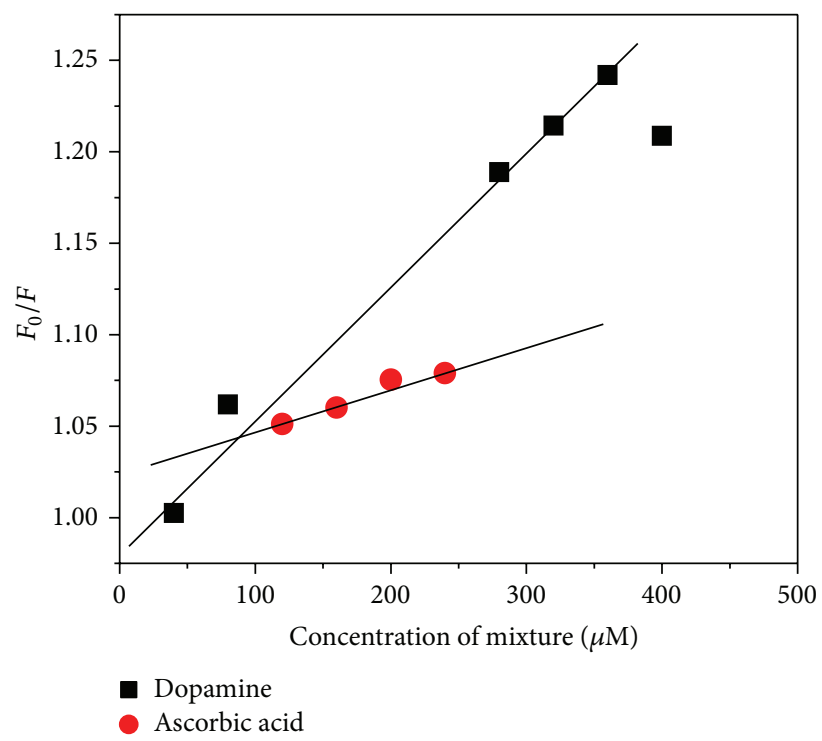

(b)

FIGURE 5: (a) The stacked photoluminescence (PL) spectra of CDs upon addition of different concentrations of a mixture of dopamine and ascorbic acid. (b) The linear fitted results for different concentrations of a mixture of dopamine and ascorbic acid showing two different linear fitting patterns.

are present together, the quenching dynamics being different, they do not interfere with each other.

Further evidence for interaction of carbon dots and dopamine can be drawn from the results obtained from zeta potential measurements. As discussed previously, zeta potential of CDs was measured to be $-184 \mathrm{mV}$ indicating the negative charge on the CDs. However, upon addition of dopamine, the value of zeta potential was found to decrease to $-56.6 \mathrm{mV}$. This clearly indicates that there is some electrostatic interaction between the negatively charged carbon dots and the amine group of dopamine molecules which are protonated in the acidic medium of the CD solution. Similar measurements with ascorbic acid did not however result in a significant change in zeta potential of the original CDs and the value obtained was $-151 \mathrm{mV}$. These results are in good agreement with the quenching efficiencies of dopamine and ascorbic acid obtained from the Stern-Volmer equation and justify the selectivity and sensitivity of the CDs towards dopamine.

The mechanism of PL emission from CDs is still a matter of debate. A number of factors contributing to $\mathrm{CD}$ luminescence are recombination of photogenerated electronhole pairs taking place on the surface of the CDs, excitons of carbon, the presence of emissive traps, the quantumconfinement effect, and so forth [18]. It is now a wellestablished fact that the interaction of molecules or ions with the surface groups of the CDs can affect the efficiency of the recombination of electron-hole [19]. Among the well-known quenching mechanisms are inner filter effects, nonradiative recombination pathways, and electron transfer processes, whereas enhancement of photoluminescence has been primarily attributed to passivation of trap states on the surface [20-24]. In our case, when surface modification is carried out (i.e., capping of CDs) emission quenching is observed which can be attributed to nonradiative recombination pathway. In our case, the irradiation of the CD solution in the absence of electron donors or acceptors results in the creation of photogenerated electron-hole pairs whose radiative recombination results in the emission of fluorescence as shown in Scheme 2.

Dopamine, being a catecholamine, acts as a fluorescence quencher of the CDs. The probable mechanisms for fluorescence quenching can be either oxidation of the hydroxyl groups of the catechol part of dopamine serving as hole traps resulting in nonradiative recombination of electron-hole and hence quenching the fluorescence of CDs or protonated amine groups (in the acidic medium of CDs solution) of dopamine acting as electron acceptors thereby preventing them from participating in radiative recombination and hence quenching the fluorescence of CDs.

\section{Conclusion}

In this work we successfully demonstrated the potential use of carbon dots prepared from tea as a fluorescent sensor for detection of dopamine. The fluorescence quenching of carbon dots was studied in presence of dopamine and ascorbic 

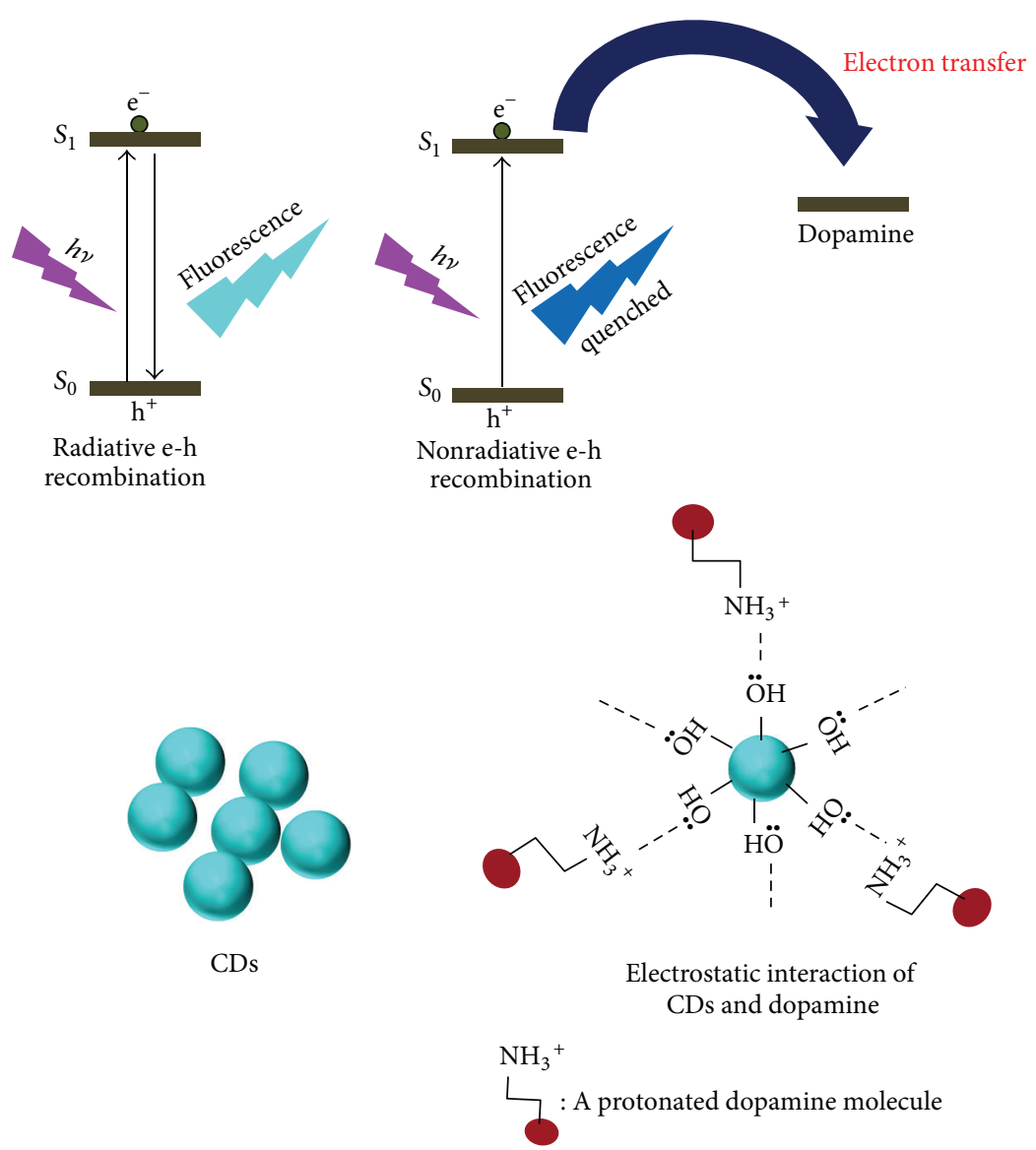

recombination

Scheme 2: A probable mechanistic representation of the fluorescence quenching of carbon dots by dopamine.

acid. From the results, it is evident that both dopamine and ascorbic acid could quench the fluorescence of CDs with dopamine showing higher quenching efficiency compared to ascorbic acid. A plausible mechanistic representation of the quenching process was also discussed. Therefore, the work clearly illustrates a simple, facile, and green method for selective and sensitive detection of dopamine and acetic acid.

\section{Conflict of Interests}

The authors declare that there is no conflict of interests regarding the publication of this paper.

\section{References}

[1] Y.-P. Sun, B. Zhou, Y. Lin et al., "Quantum-sized carbon dots for bright and colorful photoluminescence," Journal of the American Chemical Society, vol. 128, no. 24, pp. 7756-7757, 2006.

[2] X. Wang, L. Cao, F. Lu et al., "Photoinduced electron transfers with carbon dots," Chemical Communications, no. 25, pp. 37743776, 2009.

[3] S. Sahu, B. Behera, T. K. Maiti, and S. Mohapatra, "Simple onestep synthesis of highly luminescent carbon dots from orange juice: application as excellent bio-imaging agents," Chemical Communications, vol. 48, no. 70, pp. 8835-8837, 2012.
[4] M. P. Sk, A. Jaiswal, A. Paul, S. S. Ghosh, and A. Chattopadhyay, "Presence of amorphous carbon nanoparticles in food caramels," Scientific Reports, vol. 2, article 383, 2012.

[5] L. Zhu, Y. Yin, C.-F. Wang, and S. Chen, "Plant leaf-derived fluorescent carbon dots for sensing, patterning and coding," Journal of Materials Chemistry C, vol. 1, no. 32, pp. 4925-4932, 2013.

[6] K. Qu, J. Wang, J. Ren, and X. Qu, "Carbon dots prepared by hydrothermal treatment of dopamine as an effective fluorescent sensing platform for the label-free detection of iron(III) ions and dopamine," Chemistry-A European Journal, vol. 19, no. 22, pp. 7243-7249, 2013.

[7] A. Jaiswal, S. S. Ghosh, and A. Chattopadhyay, "One step synthesis of C-dots by microwave mediated caramelization of poly(ethylene glycol)," Chemical Communications, vol. 48, no. 3, pp. 407-409, 2012.

[8] A. Sachdev, I. Matai, S. U. Kumar, B. Bhushan, P. Dubey, and P. Gopinath, "A novel one-step synthesis of PEG passivated multicolour fluorescent carbon dots for potential biolabeling application," RSC Advances, vol. 3, no. 38, pp. 16958-16961, 2013.

[9] D. Chowdhury, N. Gogoi, and G. Majumdar, "Fluorescent carbon dots obtained from chitosan gel," RSC Advances, vol. 2, no. 32, pp. 12156-12159, 2012.

[10] A. Konwar, N. Gogoi, G. Majumdar, and D. Chowdhury, "Green chitosan-carbon dots nanocomposite hydrogel film with superior properties," Carbohydrate Polymers, vol. 115, no. 22, pp. 238-245, 2014. 
[11] Z. Lin, W. Xue, H. Chen, and J.-M. Lin, "Peroxynitrous-acidinduced chemiluminescence of fluorescent carbon dots for nitrite sensing," Analytical Chemistry, vol. 83, no. 21, pp. 82458251, 2011.

[12] W. Lu, X. Qin, S. Liu et al., "Economical, green synthesis of fluorescent carbon nanoparticles and their use as probes for sensitive and selective detection of mercury(II) ions," Analytical Chemistry, vol. 84, no. 12, pp. 5351-5357, 2012.

[13] U. Baruah, N. Gogoi, G. Majumdar, and D. Chowdhury, “ $\beta$ cyclodextrin and calix[4]arene-25,26,27,28-tetrol capped carbondots for selective and sensitive detection of fluoride," Carbohydrate Polymers, vol. 117, pp. 337-383, 2015.

[14] U. Baruah, N. Gogoi, G. Majumdar, and D. Chowdhury, "Capped fluorescent carbon dots for detection of hemin: role of number of -OH groups of capping agent in fluorescence quenching," The Scientific World Journal, vol. 2013, Article ID 529159, 9 pages, 2013.

[15] J. Zhao, W. Zhang, P. Sherrell et al., "Carbon nanotube nanoweb-bioelectrode for highly selective dopamine sensing," ACS Applied Materials and Interfaces, vol. 4, no. 1, pp. 44-48, 2012.

[16] Y. Tao, Y. Lin, J. Ren, and X. Qu, "A dual fluorometric and colorimetric sensor for dopamine based on BSA-stabilized Au nanoclusters," Biosensors and Bioelectronics, vol. 42, no. 1, pp. 41-46, 2013.

[17] X. Zhou, P. Ma, A. Wang et al., "Gold nanorod-based localized surface plasmon resonance biosensor for sensitive detection of hepatitis B virus in buffer, blood serum and plasma," Biosensors and Bioelectronics, vol. 64, pp. 404-410, 2015.

[18] Y. P. Sun, B. Zhou, Y. Lin et al., "Quantum-sized carbon dots for bright and colorful photoluminescence," Journal of the American Chemical Society, vol. 128, no. 24, pp. 7756-7757, 2006.

[19] X. Wang, L. Cao, F. Lu et al., "Photoinduced electron transfers with carbon dots," Chemical Communications, no. 25, pp. 37743776, 2009.

[20] B. Chen, Y. Yu, Z. Zhou, and P. Zhong, "Synthesis of novel nanocrystals as fluorescent sensors for $\mathrm{Hg}^{2+}$ ions," Chemistry Letters, vol. 33, no. 12, pp. 1608-1609, 2004.

[21] Y. Chen and Z. Rosenzweig, "Luminescent CdS quantum dots as selective ion probes," Analytical Chemistry, vol. 74, no. 19, pp. 5132-5138, 2002.

[22] R. Freeman, T. Finder, L. Bahshi, and I. Willner, " $\beta$ cyclodextrin-modified cdse/zns quantum dots for sensing and chiroselective analysis," Nano Letters, vol. 9, no. 5, pp. 20732076, 2009.

[23] K. M. Gattás-Asfura and R. M. Leblanc, "Peptide-coated CdS quantum dots for the optical detection of copper(II) and silver(I)," Chemical Communications, vol. 9, no. 21, pp. 26842685, 2003.

[24] A. Touceda-Varela, E. I. Stevenson, J. A. Galve-Gasión, D. T. F. Dryden, and J. C. Mareque-Rivas, "Selective turn-on fluorescence detection of cyanide in water using hydrophobic CdSe quantum dots," Chemical Communications, no. 17, pp. 19982000, 2008 . 

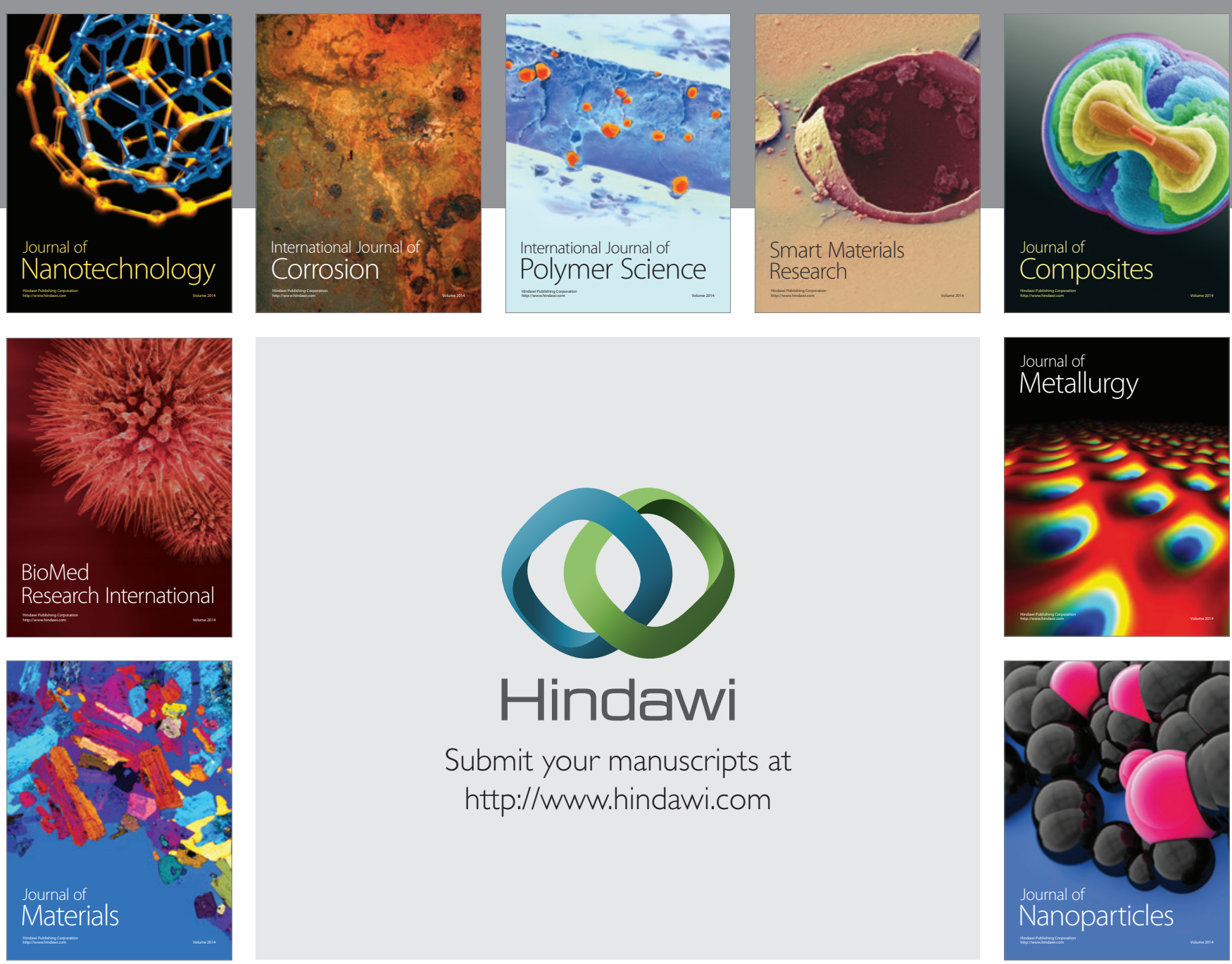

Submit your manuscripts at http://www.hindawi.com
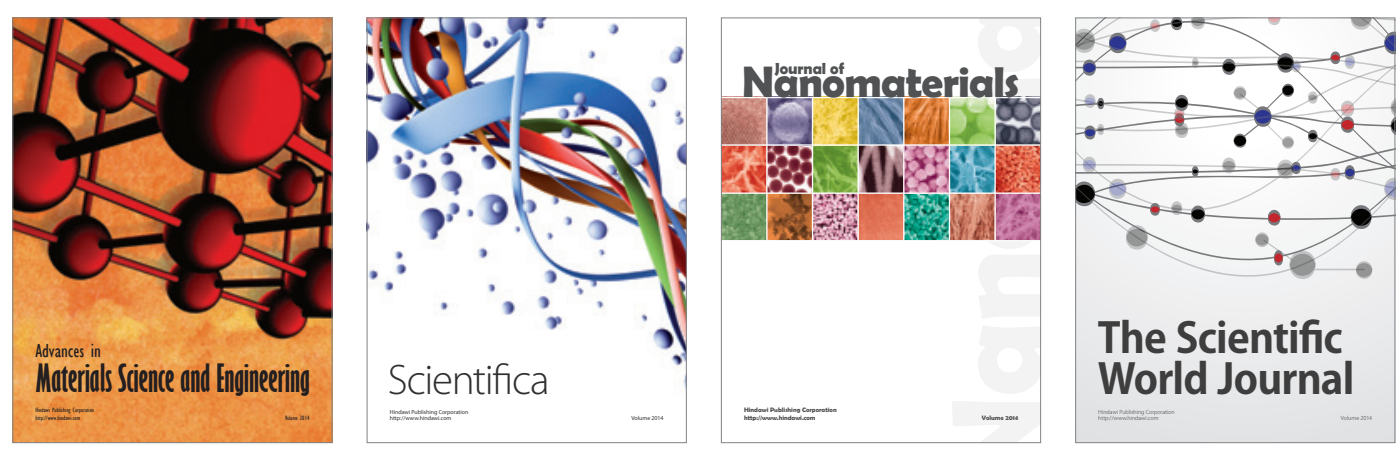

\section{The Scientific World Journal}
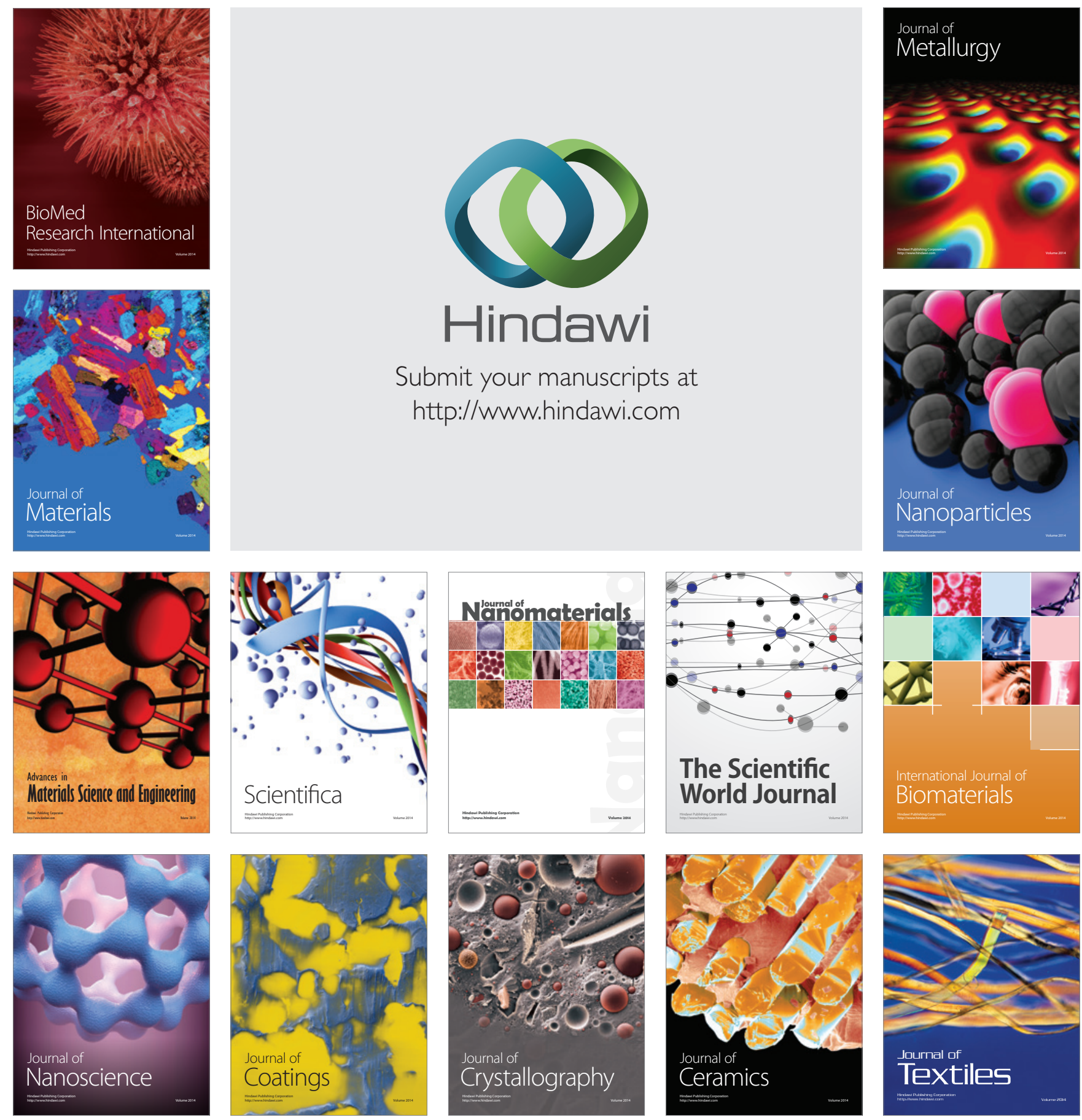